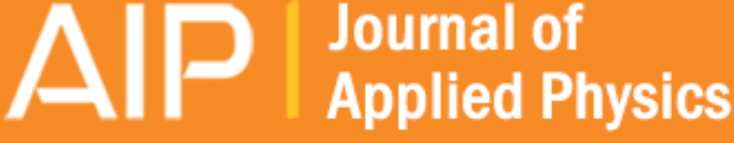

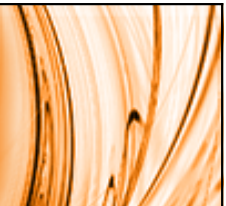

\section{Thermomagnetic energy harvesting with first order phase change materials}

Alexander Post, Chris Knight, and Erich Kisi

Citation: Journal of Applied Physics 114, 033915 (2013); doi: 10.1063/1.4815933

View online: http://dx.doi.org/10.1063/1.4815933

View Table of Contents: http://scitation.aip.org/content/aip/journal/jap/114/3?ver=pdfcov

Published by the AIP Publishing

\section{Articles you may be interested in}

Analysis of tristable energy harvesting system having fractional order viscoelastic material

Chaos 25, 013112 (2015); 10.1063/1.4905276

Empirical feasibility analysis of thermoelectric energy harvesting in thermally limited compact mobile computers J. Renewable Sustainable Energy 6, 013135 (2014); 10.1063/1.4865577

Performance of phase change materials for heat storage thermoelectric harvesting Appl. Phys. Lett. 103, 193902 (2013); 10.1063/1.4829044

Influence of electrical current variance and thermal resistances on optimum working conditions and geometry for thermoelectric energy harvesting

J. Appl. Phys. 113, 174908 (2013); 10.1063/1.4802668

Monolithic oxide-metal composite thermoelectric generators for energy harvesting

J. Appl. Phys. 109, 124509 (2011); 10.1063/1.3599890

Frustrated by

old technology?

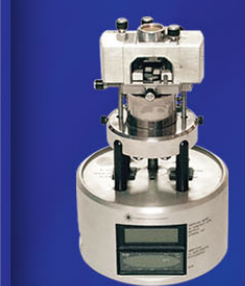

Is your AFM dead

and can't be repaired?

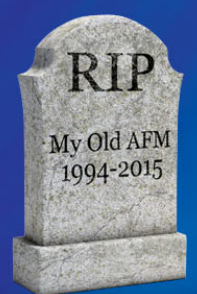

Sick of bad customer support?

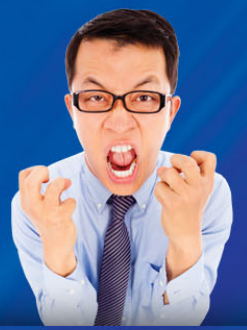

It is time to upgrade your AFM Minimum $\$ 20,000$ trade-in discount for purchases before August 31st

Asylum Research is today's technology leader in AFM 


\title{
Thermomagnetic energy harvesting with first order phase change materials
}

\author{
Alexander Post, ${ }^{1}$ Chris Knight, ${ }^{1, a)}$ and Erich $\mathrm{Kisi}^{2}$ \\ ${ }^{1}$ Commonwealth Scientific and Industrial Research Organisation (CSIRO), 10 Murray Dwyer Cct. Steel River \\ Estate, 2304, Mayfield West, NSW, Australia \\ ${ }^{2}$ School of Engineering, University of Newcastle, Newcastle, 2308, Australia
}

(Received 2 April 2013; accepted 20 June 2013; published online 19 July 2013)

\begin{abstract}
Two cycles of thermomagnetic generation have been compared, one with a conventional ferromagnetic material and one using a Heusler alloy undergoing a first order phase transformation. The two have been analytically compared in terms of overall thermal-to-magnetic energy conversion efficiency. In addition, a state-space model of a thermomagnetic generator has been developed and analyzed in order to compare the performance of the two in terms of response to decreased thermal difference and changes in plate separation. Both analytical calculations and simulation indicated a large difference in operational behavior between the two materials, with the Heusler alloy's behavior being characterized by latent heat and large changes in magnetism, and the ferromagnet being characterized by a smaller heat input and more gradual magnetic change. The theoretical energy conversion efficiency of the Heusler alloy was calculated to be $0.2 \%$ under an external field of $3 \mathrm{kOe}$, increasing to $0.8 \%$ under a field of $15 \mathrm{kOe}$. These values corresponded to $15.9 \%$ and $63.8 \%$ of the Carnot limit, respectively, and compare favorably with the conversion efficiencies of ferromagnetic materials previously used in thermomagnetic generation. (C) 2013 AIP Publishing LLC. [http://dx.doi.org/10.1063/1.4815933]
\end{abstract}

\section{INTRODUCTION}

Thermomagnetic generation of electricity relies on a change in magnetic properties that occurs with changing temperature for certain materials. If a material exhibiting thermally dependent magnetization is subject to a constant external magnetic field and cycled in temperature, its magnetization also cycles. This can interact with the external field to produce periodically cycling magnetic forces, as well as inducing its own periodically cycling magnetic field, both of which can be converted by simple means into usable electric power.

The process of thermomagnetic generation was originally proposed in the late 19 th century ${ }^{1}$ and was first analytically examined in 1948 by Brillouin et al. ${ }^{2}$ Since then it has been the subject of several studies, but has not yet been developed to a commercial level. It has been shown that the process is theoretically capable of converting power between thermal and electrical forms with relative efficiencies matching or exceeding those of Seebeck devices, ${ }^{3,4}$ yet this has not been experimentally realized.

Research into thermomagnetic generation has previously focused on the ferromagnetic family of metals and alloys at temperatures close to the Curie temperature $\left(T_{C}\right),{ }^{2,5-9}$ where a rapid loss of magnetic susceptibility causes a steep change in magnetic behavior with respect to temperature, and thus the greatest efficiency of energy conversion from thermal to magnetic. $^{10}$

Hsu et $a l .^{3}$ recently developed an expression for the theoretical maximum energy conversion efficiency of a range of polycrystalline and mono-crystalline ferromagnetic metals. The study related to the case of active magnet movement,

\footnotetext{
a) Author to whom correspondence should be addressed. Electronic mail: Chris.Knight@csiro.au.
}

and the energy output was taken as the difference in area under magnetization curves at high and low temperatures, as depicted in Figure 1. The overall efficiencies calculated by Hsu et al. ${ }^{3}$ were expressed as a fraction of the Carnot efficiency over the active range of temperature for each material. Values ranging between $2 \%$ and $65.1 \%$ of the Carnot efficiency were obtained, however the practical application of a number of these materials would be limited by Curie temperatures necessitating operation at temperatures well above or below ambient. From the materials with more accessible operating temperatures, polycrystalline Gadolinium, with a Curie temperature of $288 \mathrm{~K}$, was found by Hsu et al. ${ }^{3}$ to have a theoretical relative efficiency of $11.7 \%$ of Carnot ( $20.5 \%$ for a single crystal), and polycrystalline Nickel, with a Curie temperature of $630 \mathrm{~K}$, had a relative efficiency of $3.4 \%$. These figures were calculated with an external magnetic field strength of only $3 \mathrm{kOe}$ and were predicted to increase significantly with larger applied magnetic fields.

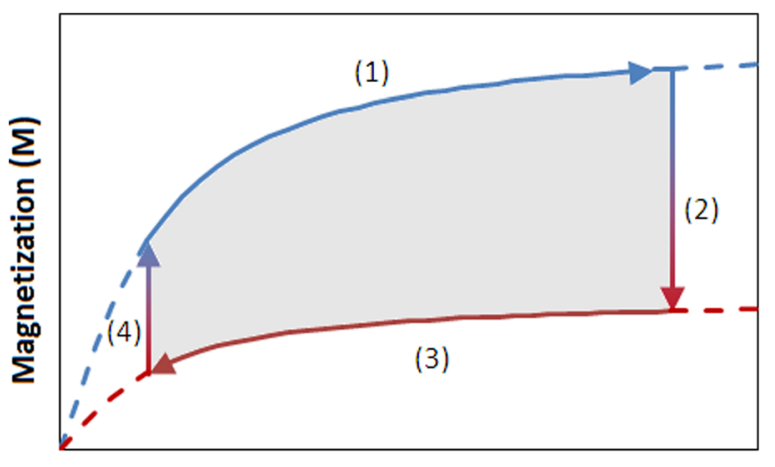

Field Strength $(\mathrm{H})$

FIG. 1. A cycle of active thermomagnetic generation, featuring magnetization (1), heating (2), demagnetization (3) and cooling (4). 
The findings of Hsu et al. ${ }^{3}$ showed that energy could be transformed between thermal and electrical forms via fluctuating fields in thermomagnetic materials with a theoretically large efficiency, in some cases much closer to the Carnot limit than that of conventional thermoelectric devices. This highlighted the potential of thermal-to-electrical energy conversion using ferromagnetic materials at the Curie temperature. However, little work has been done to examine other forms of thermally dependent magnetism in the same way.

One such form yet to be thoroughly examined is the first order transition between phases of different magnetic properties. Such a transition occurs in the Heusler alloy $\mathrm{Ni}_{45} \mathrm{Co}_{5} \mathrm{Mn}_{40} \mathrm{Sn}_{10}$, as studied by Srivastava et al., ${ }^{5,11}$ and the closely related material $\mathrm{Ni}_{44} \mathrm{Co}_{6} \mathrm{Mn}_{40} \mathrm{Sn}_{10}$, studied by Song et al. ${ }^{15}$ In the specific case of the alloy $\mathrm{Ni}_{45} \mathrm{Co}_{5} \mathrm{Mn}_{40} \mathrm{Sn}_{10}$, the material transforms from a strongly ferromagnetic austenite phase to a weakly ferromagnetic martensite phase at a critical temperature of $135^{\circ} \mathrm{C}$. Being martensitic, the phase change occurs without diffusion and with minimal movement between neighboring atoms, ${ }^{12}$ lowering the process's latent heat, and causing an abrupt change in the magnetic properties of the material over a temperature difference of only $10^{\circ} \mathrm{C}$. The particular composition was chosen by Srivastava et al..$^{5}$ for its lack of transformational stress between austenitic and martensitic phases, causing reversibility and a low thermal hysteresis. Local deviation away from this composition was shown to cause a sharp increase in thermal hysteresis, however, whether other alloy compositions exist exhibiting the same effect remains undetermined.

The significant change in the magnetic properties of the material over a low temperature difference suggests that high conversion efficiency from thermal to magnetic energy is possible, and yet a quantitative and qualitative comparison against conventional ferromagnetic materials, as conducted here, have not previously been performed. This numeric comparison will be followed by an experimental comparison conducted in Ref. 13.
Thermomagnetic generation of power has historically been accomplished in two ways; using passive and active means of magnet actuation. In this paper, both have been examined, comparing the alloy $\mathrm{Ni}_{45} \mathrm{Co}_{5} \mathrm{Mn}_{40} \mathrm{Sn}_{10}$ to the conventional ferromagnetic material gadolinium in each case.

In passive systems such as that studied by Ujihara et al., ${ }^{6}$ the cycling magnetic force produced when a thermomagnetic material is cycled in temperature is used to actuate the magnet's movement between hot and cold thermal reservoirs, which in turn perpetuates the cycling temperature, as illustrated in Figure 2.

Here, a thermomagnetic material rests in a gap between a hot magnetic reservoir and a cold non-magnetic reservoir, with a spring providing restoring force onto the cold reservoir. If the magnet is initially below its Curie temperature, it experiences a large magnetic force and travels to the hot magnetic reservoir (1). The magnet then heats up (2), losing magnetization and is pulled to the cold reservoir by the restoring spring (3). The magnet cools, regaining magnetization (4), and is attracted back to the hot reservoir, completing the cycle. Energy can be withdrawn from the system using the motion of the magnet; for instance, through the use of piezoelectric springs as proposed by Ujihara et al., ${ }^{6}$ or through inductive harvesting of the thermomagnetic material's fluctuating magnetic field.

In active systems such as that studied by Solomon, ${ }^{7}$ an external force actively moves the magnet between hot and cold thermal reservoirs while the strength of an external magnetic field is cycled, as depicted in Figure 1. The different magnetization response of the material when it is at different temperatures then causes a net transfer from thermal energy (the heat input from the hot reservoir) to magnetic energy (the secondary field created by the magnetized material) over each cycle of the external field, which is harvested through magnetic induction. This net magnetic energy gain has been depicted as the grey area in Figure 1 .
1
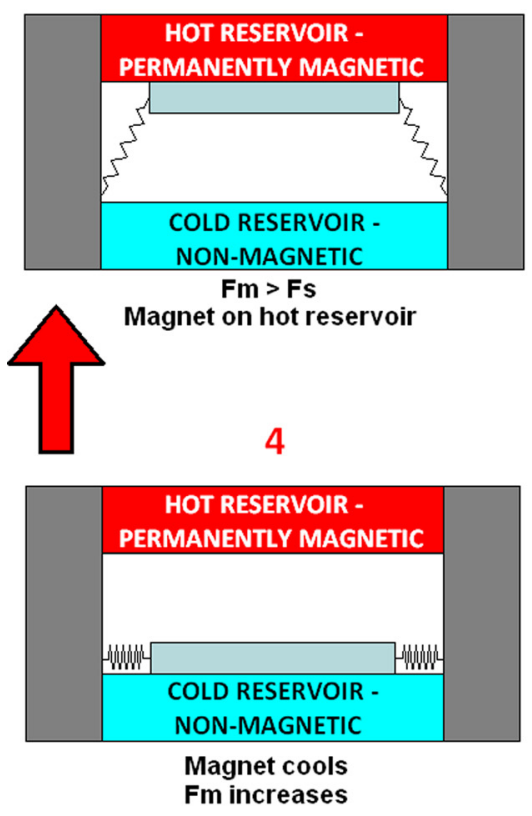

2
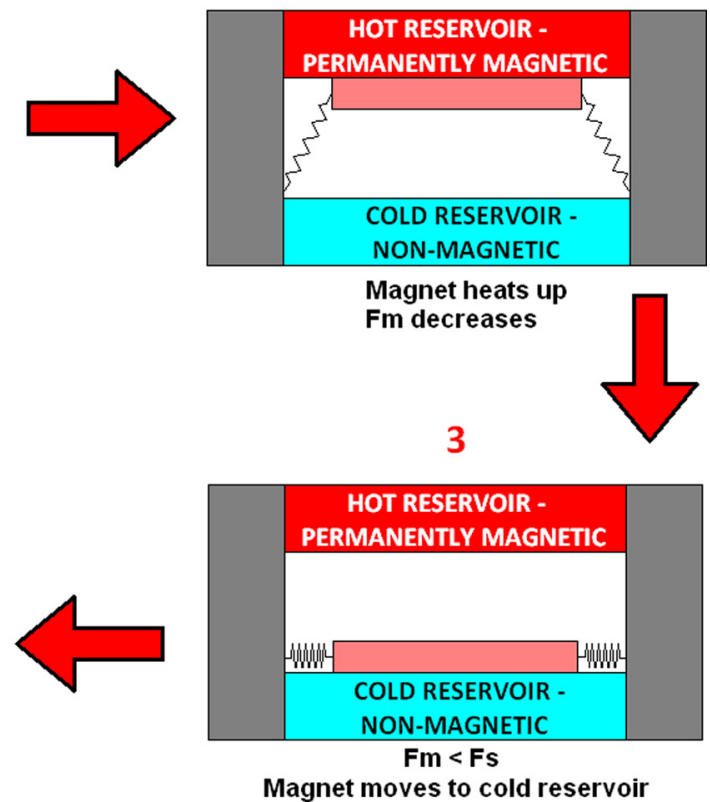

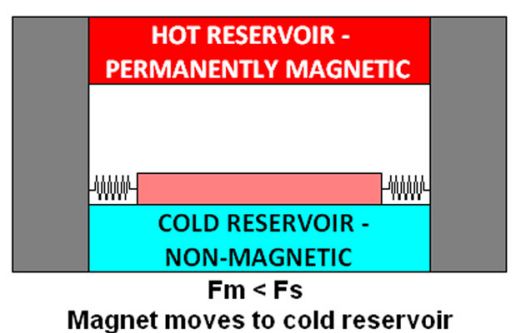

FIG. 2. Illustration of a cycle of passive thermomagnetic generation due to fluctuating magnetic force (Fm) and restoring spring force (Fs). 
Of the two, the active case typically displays higher efficiency, despite having to expend energy to actively move the magnet between reservoirs and cycle the external field (accomplished with a solenoid coil). However, the passive case can be performed by a much smaller and simpler device, requires no active control, and is self-starting when a sufficient thermal difference is applied between the hot and cold reservoirs. This study analyses device performance in both cases.

The process outlined by Hsu et al. ${ }^{3}$ has been used here to calculate an upper bound for the thermal-to-magnetic conversion efficiency of the material presented by Srivastava et $a l .{ }^{5}$ under a cycle of active thermomagnetic generation. A computer simulation has also been developed to compare the performance of this alloy with a conventional ferromagnetic material in the passive case.

\section{THEORETICAL EXAMINATION OF THE ACTIVE ACTUATION CASE}

The cycle of active thermomagnetic generation explored by Solomon ${ }^{7}$ can be described by four discrete processes: isothermal magnetization from $\mathrm{H}_{1}$ to $\mathrm{H}_{2}$ at $\mathrm{T}=\mathrm{T}_{\text {cold }}$ (stage 1 in Figure 1), heating from $\mathrm{T}_{\text {cold }}$ to $\mathrm{T}_{\text {hot }}$ with constant external field strength $\mathrm{H}_{2}$ (stage 2), isothermal demagnetization from $\mathrm{H}_{2}$ to $\mathrm{H}_{1}$ at $\mathrm{T}=\mathrm{T}_{\text {hot }}$ stage 3 ) and cooling from $\mathrm{T}_{\text {hot }}$ to $\mathrm{T}_{\text {cold }}$ with constant external field strength $\mathrm{H}_{1}$ (stage 4).

The net gain in energy of a voltage source during this cycle is described by Solomon ${ }^{7}$ as the area bounded by the curves of magnetization and demagnetization at $\mathrm{T}_{\text {hot }}$ and $\mathrm{T}_{\text {cold }}$, and can be calculated using

$$
W_{\text {out }}=\mu_{0} \oint M(H, T) d H,
$$

in which $\mu_{0}$ is the constant for vacuum permeability $\left(4 \pi \times 10^{-7}\right.$ V.s/A.m), and $\mathrm{M}$ is the magnetization of the material in Amperes per meter; a function of both temperature $\mathrm{T}$ in Kelvin and field strength $\mathrm{H}$, also in Amperes per meter. Here, $\mathrm{M}(\mathrm{H}, \mathrm{T})$ has been solved numerically using the data published by Srivastava et al. ${ }^{5}$ Since the phase change of the material takes place during the constant field strength heating and cooling stages of the cycle (stages 2 and 4 in Figure 1), the material can be treated as a single phase for stages 1 and 3 of the cycle, with magnetic susceptibilities corresponding to those of the austenite or martensite phase, respectively.

The total energy input during one cycle of the system is the sum of the thermal energy input and the magnetization energy and is presented by Hsu et al. ${ }^{3}$ as

$$
Q_{\text {in }}=\rho \int_{T_{\text {cold }}}^{T_{\text {hot }}} C_{P}(T) d T+T \int d S_{m}+\mu_{0} \oint M(H, T) d H,
$$

in which $\rho$ is the material density, $\mathrm{C}_{\mathrm{P}}$ is the material total heat capacity (in this case consisting of both simple and latent heat), and $\mathrm{S}_{\mathrm{m}}$ is the entropy of magnetization.

For low fields, the energy input of magnetization entropy becomes negligible, so the total efficiency as a ratio of input to output energy becomes

$$
\eta=\frac{\mu_{0} \oint M(H, T) d H}{\rho \int_{T_{\text {cold }}}^{T_{\text {hot }}} C_{P}(T) d T+\mu_{0} \oint M(H, T) d H} .
$$

This can be expressed as a relative fraction of the Carnot efficiency - the maximum efficiency attainable for a thermal cycle operating between cold and hot reservoirs of temperatures $\left(\mathrm{T}_{\mathrm{C}}\right)$ and $\left(\mathrm{T}_{\mathrm{H}}\right)$, respectively

$$
\eta_{r e l}=\frac{\eta}{1-\frac{T_{C}}{T_{H}}}
$$

where $T_{C}$ and $T_{H}$ are measured in degrees Kelvin.

A theoretical cycle has been considered between temperatures of $130{ }^{\circ} \mathrm{C}$ and $135^{\circ} \mathrm{C}$, as these encompass the critical phase change temperature of the material while matching the temperature gap of $5^{\circ} \mathrm{C}$ used by Hsu et al. ${ }^{3}$ The cycle was considered with a modest external field ranging from 0kOe to $3 \mathrm{kOe}$, once again to match that used by Hsu et al. ${ }^{3}$ for the purpose of comparison. The latent heat of the material was presented by Srivastava et al. ${ }^{5}$ as $132 \mathrm{~J} / \mathrm{cm}^{3}$. For a metal just above the Debye temperature, sensible heat is typically $26 \mathrm{~J} / \mathrm{mol} . \mathrm{K}$. Using this as a conservative estimate of the material's sensible heat over the range of temperatures examined gives a sensible heat input of $16.7 \mathrm{~J} / \mathrm{cm}^{3}$, and a total thermal energy input of $148.7 \mathrm{~J} / \mathrm{cm}^{3}$ per cycle.

Using Eqs. (1)-(3) and the data presented by Srivastava et al., ${ }^{5}$ the overall efficiency was calculated to be $0.2 \%$, or $15.9 \%$ of the Carnot efficiency.

The presence of a first order phase change in the material caused the thermal energy requirement in the vicinity of the critical point to be dominated by latent heat. This led to a larger input energy per cycle than that of the materials examined by Hsu et al., ${ }^{3}$ which were governed only by sensible heat. Despite this, the relative efficiencies calculated were close to those of the other polycrystalline ferromagnetic materials calculated, suggesting that the output of magnetic energy per cycle should also be large.

Upon increasing the magnetic field strength applied during a cycle, the input thermal energy remains constant and the output magnetic energy increases. Thus, due to the large difference in magnetization of this material at temperatures above and below the critical temperature, it is likely that the efficiency of this harvester would increase significantly with a larger applied magnetic field. Repeating the above calculation with an applied field cycling between $0 \mathrm{kOe}$ and $15 \mathrm{kOe}$ (approximately that found at the surface of an $\mathrm{NdFeB}$ permanent magnet), the calculated efficiency increased to $0.8 \%$, corresponding to a relative efficiency of $63.8 \%$ of the Carnot limit-significantly higher than that of modern thermoelectrics. However, the accuracy of this figure may be impacted upon by the omission of the magnetic entropy term in Eq. (2), which becomes significant for applied magnetic fields larger than $10 \mathrm{kOe}$.

This result correlates with those published in Figure 9 of Song et al., ${ }^{15}$ in which the effect of increasing the range of field strengths $(\Delta \mathrm{H})$ over a cycle was found to substantially increase the energy conversion efficiency for two different 
thermodynamic cycles with the phase changing alloy $\mathrm{Ni}_{45} \mathrm{Co}_{5} \mathrm{Mn}_{40} \mathrm{Sn}_{10}$. These figures also compare favorably to the relative efficiencies of $11.4 \%$ of Carnot under $3 \mathrm{kOe}$ and $\approx 20 \%$ of Carnot under $15 \mathrm{kOe}$ for polycrystalline Gadolinium found in Hsu et al. ${ }^{3}$

\section{NUMERICAL ANALYSIS OF THE PASSIVE ACTUATION CASE}

In the process of passive thermomagnetic generation, the cycle speed and movement of the system are not externally controlled, but instead allowed to naturally equilibrate. Thus, the system is difficult to examine in terms of energy output and efficiency purely by analytic means. In this study, the system has been studied through the use of a state-space model in terms of heat flow and force balance equations, from which the rates of input and output energy have been approximated. Cycles were simulated with discs of Gadolinium; a conventional ferromagnetic material frequently studied for use in thermomagnetic applications, ${ }^{6,7,9}$ and the alloy $\mathrm{Ni}_{45} \mathrm{Co}_{5} \mathrm{Mn}_{40} \mathrm{Sn}_{10}$, in order to compare the behavior of the two.

The system considered here is a typical cycle of passive thermomagnetic generation, as studied by Srivastava et al. ${ }^{5}$ and Bulgrin et al., ${ }^{8}$ featuring a disc of magnetic material situated between a hot magnetic reservoir and a cold nonmagnetic reservoir, with a piezoelectric spring providing restoring force onto the cold reservoir and a means to withdraw mechanical energy from the system. However, in the case of the $\mathrm{Ni}_{45} \mathrm{Co}_{5} \mathrm{Mn}_{40} \mathrm{Sn}_{10}$ alloy, the magnetic phase occurs at a higher temperature than the non-magnetic phase, so the hot and cold reservoirs have been reversed (with the spring providing a restoring force onto a hot non-magnetic reservoir).

Bulgrin et al. ${ }^{8}$ derived an expression for the magnetic force acting on a gadolinium disc in a static magnetic field using a numeric fit of experimental data. Here, this expression has been used to relate the magnetic force acting on the thermomagnetic disc to the disc's temperature and separation from the permanent magnet, providing a link between thermal and mechanical subsystems, as in the following equation:

$$
F_{M}=\frac{m}{0.07} e^{\frac{-1000 y}{0.55}}\left(1383 e^{\frac{-T}{43}}-341\right)+223 e^{\frac{-T}{24.3}}-21.6 .
$$

The magnetic force in this expression is a function of both the temperature of the disc $(T)$ in Kelvin and its separation from the permanent magnet $(y)$ in meters, as well as the magnet mass $(m)$ in $\mathrm{kg}$.

A similar expression was derived for a disc of $\mathrm{Ni}_{45} \mathrm{Co}_{5} \mathrm{Mn}_{40} \mathrm{Sn}_{10}$ using the method described by Carr, ${ }^{14}$ in which the overall magnetization of a multi-phase material may be calculated as the sum of the individual magnetizations its component phases, weighted by mass. This was multiplied by the temperature dependent term in Eq. (5), resulting in the following equation:

$$
F_{M}=\frac{m}{0.07} e^{\frac{-1000 y}{0.55}}\left(M_{M}\left(1-F_{A}\right)+M_{A}\left(F_{A}\right)\right),
$$

in which $\mathrm{M}_{\mathrm{M}}$ and $\mathrm{M}_{\mathrm{A}}$ are the respective magnetizations of the martensite and austenite phases, and $\mathrm{F}_{\mathrm{A}}$ the fraction of austenite present in the sample at any given temperature, as described by

$$
F_{A}=\operatorname{erf}(0.382(T-150.342)) 0.5+0.5,
$$

in which erf denotes the mathematical error function. This expression was determined from a least squares fit of the magnetization data for $\mathrm{Ni}_{45} \mathrm{Co}_{5} \mathrm{Mn}_{40} \mathrm{Sn}_{10}$ published by Srivastava et al. ${ }^{5}$ A thermal hysteresis of $132 \mathrm{~J} / \mathrm{cm}^{3}$ was also incorporated into the model to account for latent heat. Thus over a cycle, the total heat input will equal the latent heat plus the sensible heat corresponding to $\Delta \mathrm{T}$. An additional allowance has to be made for the influence of field strength on the transition temperature, as predicted by the ClausiusClapeyron equation, due to the change in magnetic field strength between the hot and cold reservoirs. In this case, the increase in transition temperature at the hot reservoir has been estimated to be $1.9 \mathrm{~K}$, for a $0.5 \mathrm{~T}$ difference (extracted from Figure 2 of Srivastava et $\mathrm{al}^{11}{ }^{11}$ ). This has been added to the hysteresis due to latent heat in the simulation.

The thermal system was modelled using a lumped capacitance approximation, with heat conduction across the contact resistance $\mathrm{R}_{\mathrm{c}}{ }^{\prime \prime}$ given by Newton's law of cooling occurring at the reservoirs, and adiabatic behaviour assumed during magnet transitions.

The basic model parameters used in the simulation have been listed in Table I.

The motion of each disc was simulated over a $10 \mathrm{~s}$ period, along with the disc's temperature, magnetic force, and in the case of the multi-phase alloy, the material's phase composition. As can be seen from Figure 3, pronounced fluctuations of temperature and magnetic force occurred during each cycle for the gadolinium disc.

The temperature of the disc was observed to fluctuate between $15.3^{\circ} \mathrm{C}$ and $33.4^{\circ} \mathrm{C}$, at which points the magnetization of the disc passed zero net force, causing the disc to move between reservoirs. Discrete jumps were observed in the magnetic force due to changing displacement between the disc and the permanent magnet, separated by gradual changes in magnetism caused by changing disc temperature. A similar cycle for the Heusler alloy $\mathrm{Ni}_{45} \mathrm{Co}_{5} \mathrm{Mn}_{40} \mathrm{Sn}_{10}$ is depicted in Figure 4.

TABLE I. State-space model parameters.

\begin{tabular}{lccc}
\hline \hline Parameter & Symbol & Value & Units \\
\hline Magnet mass & $M$ & $1.24 * 10^{-3}$ & $\mathrm{~kg}$ \\
Magnet cross section & $A$ & $3.14 * 10^{-4}$ & $\mathrm{~m}^{2}$ \\
Magnet height & $L$ & $5 * 10^{-4}$ & $\mathrm{~m}$ \\
Magnet stroke & $\Delta y$ & $5 * 10^{-4}$ & $\mathrm{~m}$ \\
Spring natural position & $y_{0}$ & $5 * 10^{-2}$ & $\mathrm{~m}$ \\
Spring constant & $K$ & 100 & $\mathrm{~N} / \mathrm{m}$ \\
Cold reservoir temperature ${ }^{\mathrm{a}}$ & $T_{c}$ & $0 / 120$ & ${ }^{\circ} \mathrm{C}$ \\
Hot reservoir temperature $^{\mathrm{a}}$ & $T_{h}$ & $50 / 170$ & ${ }^{\circ} \mathrm{C}$ \\
Contact resistance & $R_{c}{ }^{\prime \prime}$ & $1.67 * 10^{-3}$ & $\mathrm{~m}^{2} \cdot \mathrm{K} / \mathrm{W}$ \\
Magnet specific heat $^{\mathrm{a}}$ & $C_{p}$ & $235.9 / 253.3$ & $\mathrm{~J} / \mathrm{kg} \cdot \mathrm{K}$ \\
\hline \hline
\end{tabular}

${ }^{\mathrm{a}}$ Where different simulation parameters have been used for a different material, values have been quoted as (value for ferromagnet)/(value for $\mathrm{Ni}_{45} \mathrm{Co}_{5} \mathrm{Mn}_{40} \mathrm{Sn}_{10}$ ). 

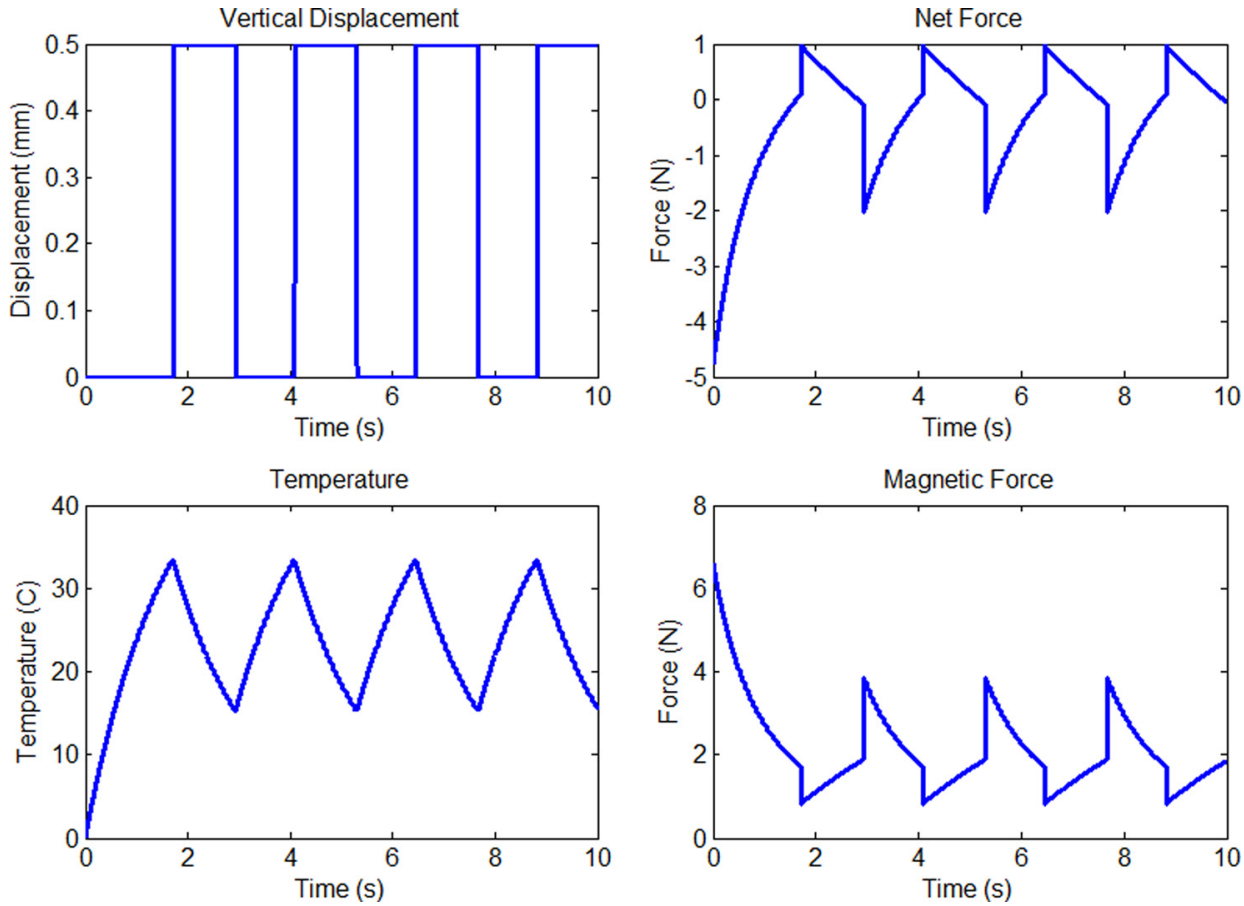

FIG. 3. Simulated passive cycle for gadolinium in terms of displacement, temperature, net, and magnetic forces.

Several differences were observed between the behavior of a harvester using $\mathrm{Ni}_{45} \mathrm{Co}_{5} \mathrm{Mn}_{40} \mathrm{Sn}_{10}$ and a conventional ferromagnet. Since the thermal behavior of the alloy was latent heat dominated, only a slight oscillation in temperature occurred over each cycle, with maximum and minimum temperatures of $150.5^{\circ} \mathrm{C}$ and $148.5^{\circ} \mathrm{C}$ respectively. This was caused by the material's much sharper magnetic response to temperature, and meant that heat flow would not diminish as the temperature of the disc approached that of the reservoir. Instead of oscillating in temperature, the material oscillated between phases, causing a corresponding oscillation in magnetic force on the disc and plateaus in temperature as the latent heat was added to or withdrawn from the material.
The phase composition of the alloy was observed to vary between $16.3 \%$ and $53.6 \%$ Austenite, again corresponding to points of zero net force, and causing the temperature of the disc to fluctuate about a point higher than the critical temperature of $130^{\circ} \mathrm{C}$. The abrupt change in magnetism accompanying the phase change caused the $\mathrm{Ni}_{45} \mathrm{Co}_{5} \mathrm{Mn}_{40} \mathrm{Sn}_{10}$ disc to oscillate more rapidly despite its latent heat requirement, with a frequency of $0.76 \mathrm{~Hz}$ exceeding that of $0.42 \mathrm{~Hz}$ for the gadolinium system.

Frequency, output power and efficiency were calculated from the simulation using the cycle time, input thermal energy and output mechanical work (the product of spring force and displacement). By repeating these simulations with
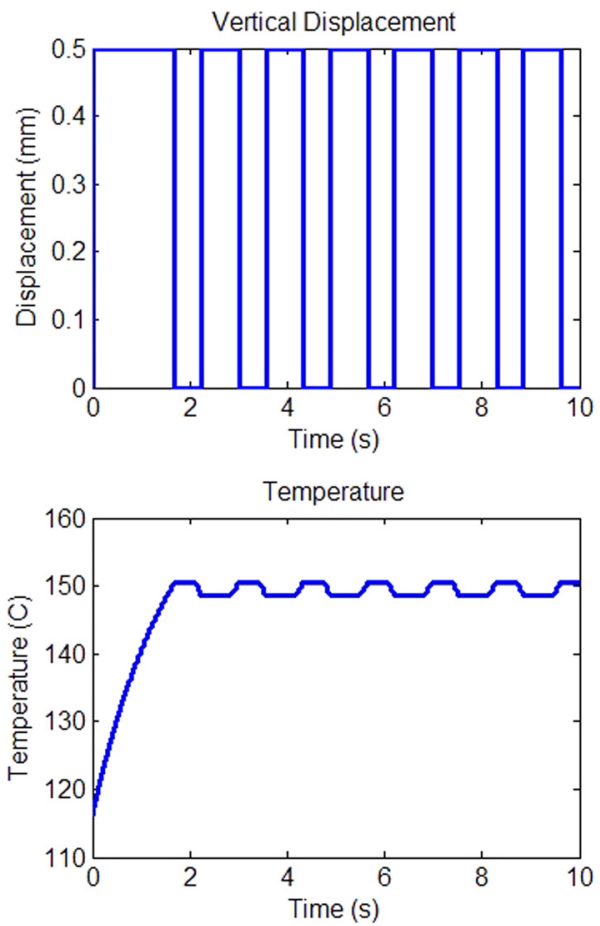
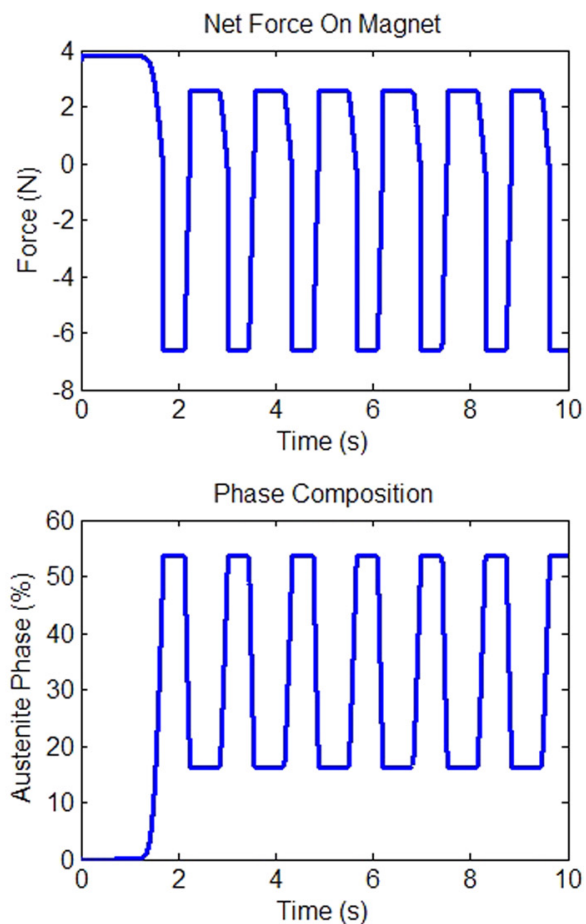

FIG. 4. Simulated passive cycle for $\mathrm{Ni}_{45} \mathrm{Co}_{5} \mathrm{Mn}_{40} \mathrm{Sn}_{10}$ in terms of displacement, temperature, net force and phase composition. 

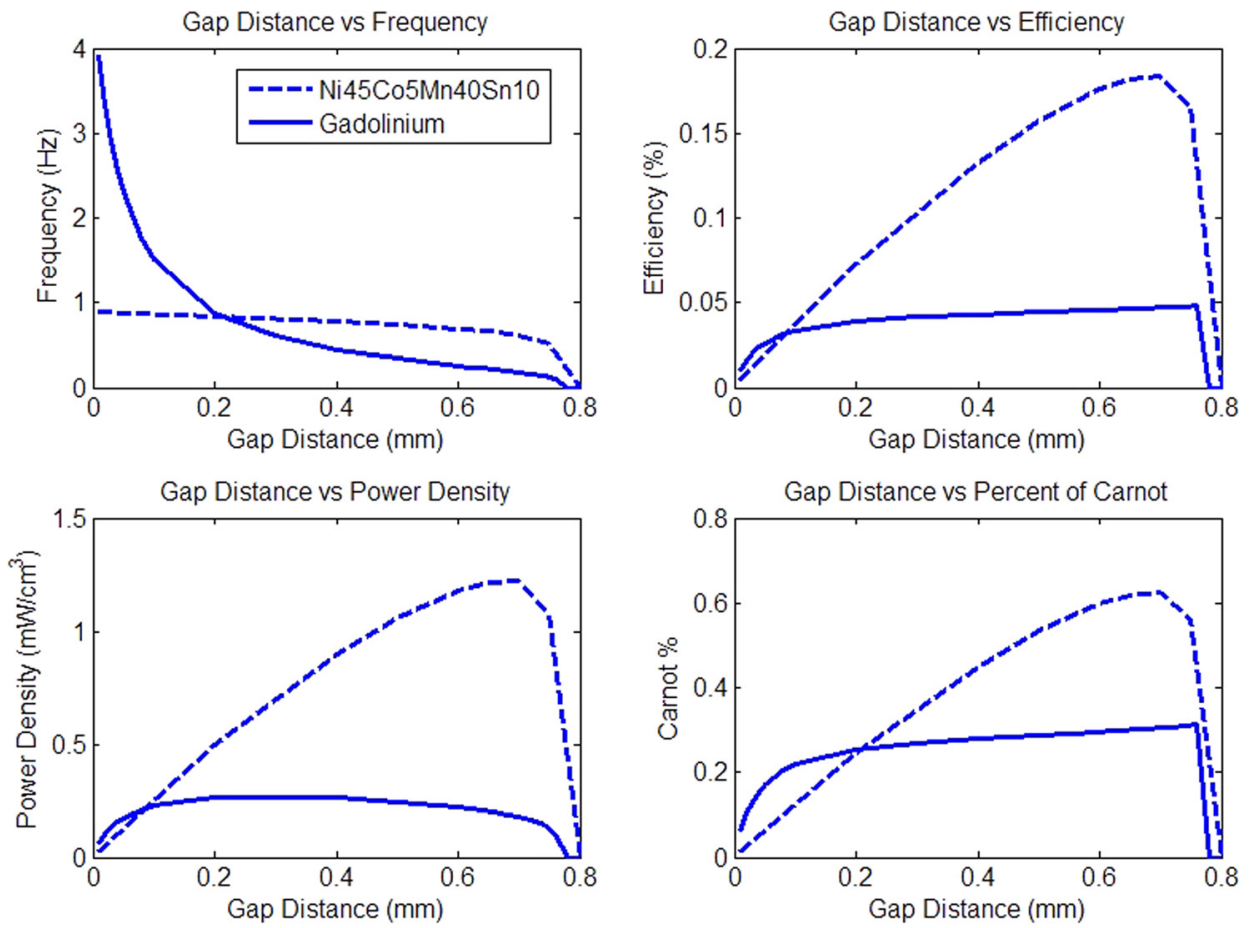

FIG. 5. Simulation response to plate separation. different parameters, the effects of changing gap distance and reservoir temperature on the performance of both systems were observed.

The simulated responses of both materials to changing reservoir separation are contained in Figure 5.

In these calculations, the gap distance was taken as the distance travelled by the magnetic disc during an oscillationi.e., the distance between hot and cold reservoirs minus the thickness of the disc. As can be seen from Figure 5, varying this parameter had a strong effect on the performance of both devices. In agreement with the previously published work of Ujihara et al. ${ }^{6}$ and Bulgrin et al., ${ }^{8}$ an exponential increase in frequency was observed for the Gadolinium harvester with decreasing gap distances, while output power peaked with a gap distance of $0.2 \mathrm{~mm}$, decreasing for smaller gap distances due to a shorter distance over which mechanical work was performed, and for larger gap distances due to a frequency tending towards zero.

Meanwhile, the frequency response of the multi-phase alloy $\mathrm{Ni}_{45} \mathrm{Co}_{5} \mathrm{Mn}_{40} \mathrm{Sn}_{10}$ was comparatively stable over the range of gap distances observed, remaining between $1.15 \mathrm{~Hz}$ and $0.85 \mathrm{~Hz}$ for distances ranging from 0 to $0.6 \mathrm{~mm}$. Such behavior exemplifies the influences of latent heat input and hysteresis on device dynamics. The difference between the magnetizations of austenitic and martensitic phases in the sample create a rapidly changing magnetic force, while the hysteresis accompanying the second order phase change causes a lag in thermal input. The result of this is an increased dependence on heat flow and a decreased dependence on device geometry.

Both materials displayed local maxima in power density (per total volume of harvester) and efficiency with respect to gap distance, the Gadolinium harvester achieving a maximum power density of $0.27 \mathrm{~mW} / \mathrm{cm}^{3}$ with a $0.3 \mathrm{~mm}$ gap and maximum efficiency of $0.05 \%$ with a $0.76 \mathrm{~mm}$ gap, while the
$\mathrm{Ni}_{45} \mathrm{Co}_{5} \mathrm{Mn}_{40} \mathrm{Sn}_{10}$ achieved maxima in both power density of $1.22 \mathrm{~mW} / \mathrm{cm}^{3}$ and efficiency of $0.18 \%$ with a $0.65 \mathrm{~mm}$ gap. Qualitatively, the fraction of Carnot efficiency was the same as the overall efficiency with respect to gap distance for both harvesters due to the constant reservoir temperatures. However, the gadolinium harvester's lower operating temperature lowered its Carnot limit, causing its relative efficiency to exceed that of the $\mathrm{Ni}_{45} \mathrm{Co}_{5} \mathrm{Mn}_{40} \mathrm{Sn}_{10}$ harvester for gap distances below $0.2 \mathrm{~mm}$.

Both harvesters displayed a sharp drop in both power and efficiency for gaps greater than $0.75 \mathrm{~mm}$, over which distance the magnetic force became too weak to complete a cycle, causing frequency to tend to zero.

The responses of both systems to changing reservoir temperatures are contained in Figure 6.

The effects of reservoir temperature difference were observed on both harvesters by incrementally increasing the temperature of the hot reservoir and decreasing the temperature of the cold reservoir, in such a way that a constant average was maintained between the two. This resulted in a linear increase in frequency for both harvesters, as depicted in the Frequency-Temperature plot of Figure 6, due to increased heat flow into and out of the magnetic material.

Both materials exhibited linear increases in output power and frequency with increasing temperature difference between the plates, although the rate of this was larger for the $\mathrm{Ni}_{45} \mathrm{Co}_{5} \mathrm{Mn}_{40} \mathrm{Sn}_{10}$ alloy, due to a large thermal influence on behavior. The cycle efficiency remained close to constant for both systems with respect to temperature difference, differing by less than $0.01 \%$ for each system between temperature differences of $20^{\circ} \mathrm{C}$ and $100^{\circ} \mathrm{C}$. This produced a large increase in the relative efficiency (as a percentage of the Carnot limit) of both cycles for small temperature differences, as would be expected from Equation (4) with a constant efficiency with respect to temperature. Such behavior is 

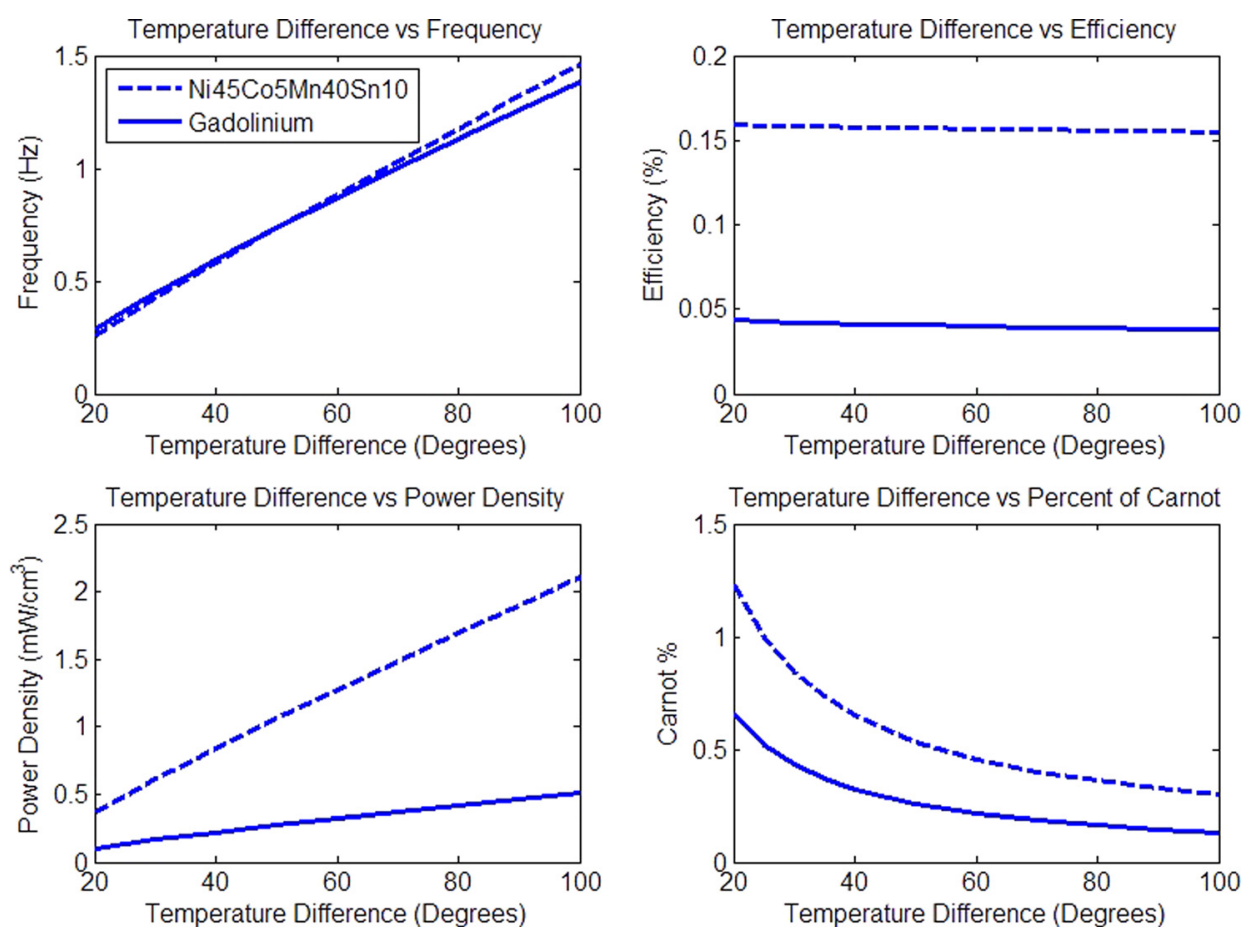

FIG. 6. Simulated reservoir temperature dependence of both systems. uncommon among thermal harvesters, in which efficiencies normally follow the same trend as the Carnot limit and decrease for decreasing temperature difference. The constant efficiencies of both harvesters over this range indicate a high potential for application in harvesting low grade heat sources.

\section{CONCLUSION}

In the cases of both active and passive magnet actuation, larger output power and efficiency were observed with a multi-phase alloy than with a conventional ferromagnet, despite the need to supply increased thermal energy due to the material's latent heat.

In the active case, the calculated relative efficiency of $15.9 \%$ of the Carnot limit exceeded that predicted for the conventional ferromagnetic material polycrystalline Gadolinium (11.7\%) in Hsu et al. ${ }^{3}$ and was predicted to increase substantially for stronger applied magnetic fields.

In the passive case, power density and efficiency were found to exceed those of Gadolinium for a large range of geometries and temperature differences, with a maximum percentage of the Carnot limit of $1.25 \%$ achieved with a temperature difference of only $20^{\circ} \mathrm{C}$. Overall conversion efficiency was found to be only weakly dependent on the temperature difference between reservoirs, showing promise for the harvesting of low grade heat sources.

In both applications the behavior of the $\mathrm{Ni}_{45} \mathrm{Co}_{5} \mathrm{Mn}_{40} \mathrm{Sn}_{10}$ alloy was characterized by states of very different magnetization, with a large energy input required to transform between them. If materials can be developed exhibiting a similar change in magnetic characteristics but a lower latent heat requirement, the power and efficiencies of these devices should greatly improve, and may surpass that of current alternatives.

It has been shown that the development of multi-phase materials such as the Heusler alloy examined here could have a number of promising effects on thermomagnetic generation, including elevated performance of small devices and a high relative efficiency over small thermal differences, as well as an increased rate of heat influx from the environment.

${ }^{1}$ N. Tesla, U.S. patent 428057 (1890).

${ }^{2}$ L. Brillouin and H. P. Iskenderian, Electron. Commun. 25, 300 (1948).

${ }^{3}$ C. Hsu, S. M. Sandoval, K. P. Wetzlar, and G. P. Carman, J. Appl. Phys. 110, 12393 (2011).

${ }^{4}$ A. V. Rosa, Fundamentals of Renewable Energy Processes (Elsevier, Oxford, 2009), p. 172.

${ }^{5}$ V. Srivastava, Y. Song, K. Bhatti, and R. D. James, Adv. Energy Mater. 1, 97-104 (2011).

${ }^{6}$ M. Ujihara, G. P. Carman, and D. G. Lee, Appl. Phys. Lett. 91, 093508 (2007).

${ }^{7}$ D. Solomon. J. Appl. Phys. 63, 915 (1988).

${ }^{8}$ K. E. Bulgrin, Y. Sungtaek, G. P. Carman, and A. S. Lavine, in Proceedings of the ASME 2009 International Mechanical Engineering Congress \& Exposition, Lake Buena Vista, Florida, USA, November 1319, 2009, pp. 327-335.

${ }^{9}$ L. D. Kirol and J. I. Mills, J. Appl. Phys. 56, 824 (1984).

${ }^{10}$ R. Bozorth, Ferromagnetism (Wiley IEEE Press, New York, 1978).

${ }^{11}$ V. Srivastava, X. Chen, and R. D. James, Appl. Phys. Lett. 97, 014101 (2010).

${ }^{12}$ S. Banerjee and P. Mukhopadhyay, Pergamon Materials Series (Elsevier, Oxford, 2010), Vol. 12, pp. 260-262.

${ }^{13}$ A. Post, C. Knight, and E. Kisi "Experimental comparison of materials for application in thermomagnetic generation," (unpublished).

${ }^{14}$ W. J. Carr, Jr., Magnetism and Metallurgy, edited by A. Berkowitz and E. Kneller (Academic Press, New York and London, 1969), Vol. 1, pp. 45-91.

${ }^{15}$ Y. Song, K. P. Bhatti, V. Srivastava, C. Leighton, and R. D. James, Energy Environ. Sci. 6, 1315 (2013). 